\title{
New aspect of hepatic nuclear glycogenosis in diabetes ${ }^{1}$
}

\author{
F. CARAMIA, F. G. GHERGO, C. BRANCIARI, AND G. MENGHINI \\ From the Institute of General Pathology, University of Rome, Italy
}

SYNOPSIS Three cases of nuclear glycogenosis in the liver of diabetic patients have been studied by electron microscopy. In addition to the glycogen deposits described by others, an unusual intranuclear glycogen-filled body was found in all three cases. This body occurred alone or in close contact with the major glycogen deposit.

Glycogen in the nucleus was originally found in the human hepatocytes. It is common in diabetes mellitus (Ehrlich, 1883; Chipps and Duff, 1942) and in von Gierke's disease (Sheldon, Silverberg, and Kerner, 1962), but also occurs in diseases such as arteriosclerosis, neoplasms, and acute suppurative inflammations (Chipps and Duff, 1942). The glycogen deposits begin to appear in the centre of the nucleus and grow concentrically so that a margin of chromatin material always separates them from the cytoplasm. In the liver nuclear glycogen has an ultrastructural appearance which differs from that of hepatic cytoplasmic glycogen, more closely resembling glycogen of skeletal muscle and other normal (Revel, Napolitano, and Fawcett, 1960) and degenerating cells (Luse, Caramia, Gerritsen, and Dulin, 1967), i.e., it is composed of small randomly-arranged particles. Glycogen in the nucleus also occurs in the liver of well-fed tadpoles during the premetamorphic stage (Himes and Pollister, 1962). Because of the different structure and central site, these authors have suggested that the glycogen in the nucleus is synthesized in situ.

In the course of electron microscopic studies of liver biopsies from patients with various diseases, we have observed in three cases of nuclear glycogenosis in diabetics a previously undescribed intranuclear structure which seems to have a distinct relation to the glycogen deposits.

\section{MATERIALS AND METHODS}

Needle biopsies of the liver by the Menghini technique

${ }^{1}$ This work was supported in part by grants from the Consiglio Nazionale delle Ricerche. It was presented in part at the European Association for the Study of the Liver, 23 and 24 April 1966, Marburg, Germany.

Received for publication 4 July 1967.
(1959) were obtained from six adult patients, three male and three female, all diabetic for 10 to 15 years and treated with insulin. The tissue was promptly immersed is osmium tetroxide $2 \%$ in $0.1 \mathrm{M}$ phosphate buffer, $p \mathrm{H} \mathrm{7.4}$, and fixed for one and a half to two hours at $4{ }^{\circ} \mathrm{C}$., then dehydrated in ethanol and embedded in Epon 812. Thin sections, cut on glass knives in a Porter-Blum microtome, were mounted on copper grids without a supporting film and stained with lead hydroxide (Millonig, 1961) or lead citrate (Renolds, 1963). Sections were examined in an RCA EMU-3D or $3 \mathrm{H}$ electron microscope. One micron sections in Epon were stained with the periodic-acid-Schiff reagent. Diastase digestion was unsuccessful in the Epon-embedded material.

\section{RESULTS}

LIGHT MICROSCOPY P.A.S.-positive deposits were present in $25 \%$ to $50 \%$ of the nuclei in three of the six cases. Such P.A.S.-positive material is found in two different forms: (1) diffusely in the nucleus and (2) as a small, densely stained round body (nuclear glycogen body) about the size of the nucleolus. Frequently the two forms are present in the same nucleus. In such nuclei the small round body always is at the periphery of the diffuse nuclear glycogen inclusion (Fig. 2). However, the small P.A.S.-dense bodies also may occur alone and then are located centrally in the nucleus (Fig. 1). Never was more than one small dense body seen in a nucleus.

ELECTRON MICROSCOPY A typical intranuclear deposit of glycogen as previously described (Sparrow and Ashworth, 1965) is shown in Figure 3. The glycogen mass is located centrally and is completely surrounded by a rim of chromatin of varying thickness which separates the glycogen from the nuclear membrane. The nucleolus is displaced to the peri- 


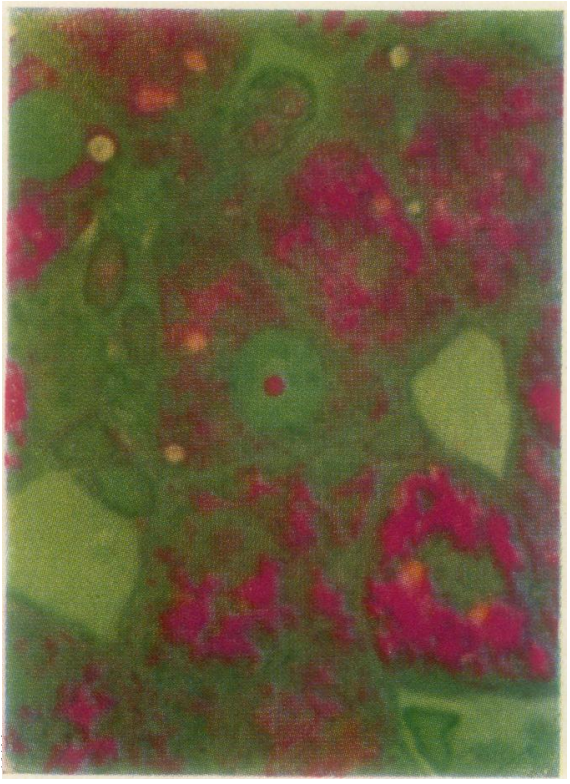

FIG. 1 .

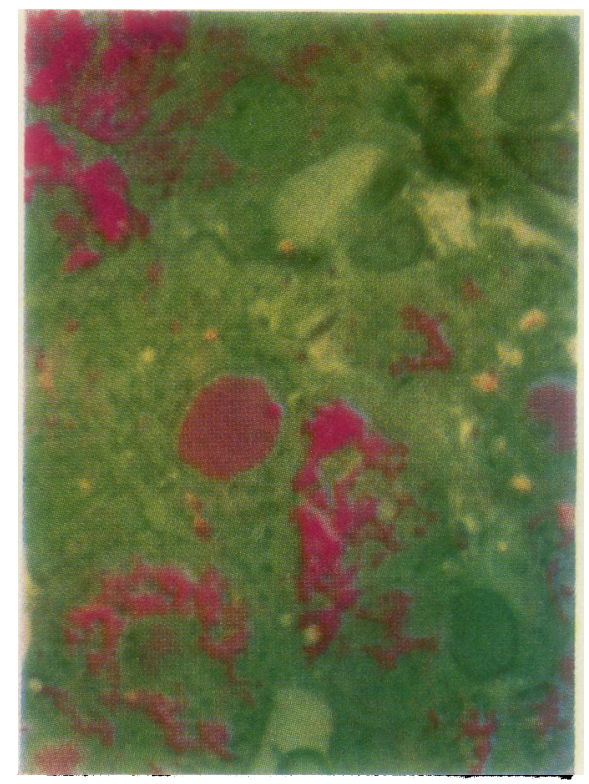

FIG. 2.

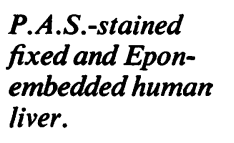

liver.

FIG. 1. This micrograph shows at high magnification a nucleus with a centrally placed small dense, P.A.S.- positive body. $\times 1,200$.

FIG. 2. A single glycogen-containing nucleus is shown at higher magnification. Almost embedded in the inclusion at one margin is a small round dense body that is intensely P.A.S.-positive. $\times 1,200$.

phery of the nucleus along with the chromatin which separates it from the glycogen deposit. Nuclear glycogen is present as single particles about $250 \AA$ in diameter which occasionally appear aggregated in small masses of varying size and shape. Cytoplasmic glycogen, by contrast, appears as uniform small rosettes ( $\alpha$ particles) as is characteristic in the human liver.

In many nuclei a round body is present (the P.A.S.positive nuclear glycogen body seen by light microscopy) which contains material intensely stained by lead surrounded by a less dense halo of uniform thickness (Fig. 4). This formation has been found in the centre of nuclei where no other glycogen deposit is present in the nucleus and where the nucleolus is not in the plane of the section. At high magnification the halo surrounding this central dense body is denser than nucleoplasm and is formed of a homogeneous finely granular material. The dense material within the central body consists of particles identical in appearance to the less densely packed glycogen elsewhere in the nucleus. This body rarely is observed in the absence of other intranuclear glycogen deposits. Usually it is located at one side of a glycogen deposit (Figs. 5 and 6). With large glycogen deposits the body is displaced to the periphery of the nucleus together with the nucleolus and chromatin
(Fig. 6). In such nuclei the peripheral halo always remains in contact with the glycogen deposit. The nucleolus, although apparently near the body and the glycogen deposit, remains separated from these structures by a layer of chromatin (Fig. 6). Never have we observed more than one of these bodies in. a nucleus. In binucleate cells, the nuclei usually ar identical; both appear normal or both contairs bodies and glycogen.

\section{DISCUSSION}

In the six cases of diabetes we have examined hepatic nuclear glycogenosis was present in three은 This finding appears neither to be related to insulin treatment nor to duration of the disease since all six patients had received insulin for the 10 to 15 years of their disease. The appearance of the nuclear glycogen resembled in part that described by previous authors. In most nuclei the inclusion of glycogen was centrally located and was not sur rounded by any membranous structure but ratheps appeared to be in direct contact with the nucleo: plasm. When large glycogen deposits were presen $\overline{6}$ the chromatin material and nucleolus were pushed peripherally; even in these nuclei the glycogen deposit did not come in direct contact with the 


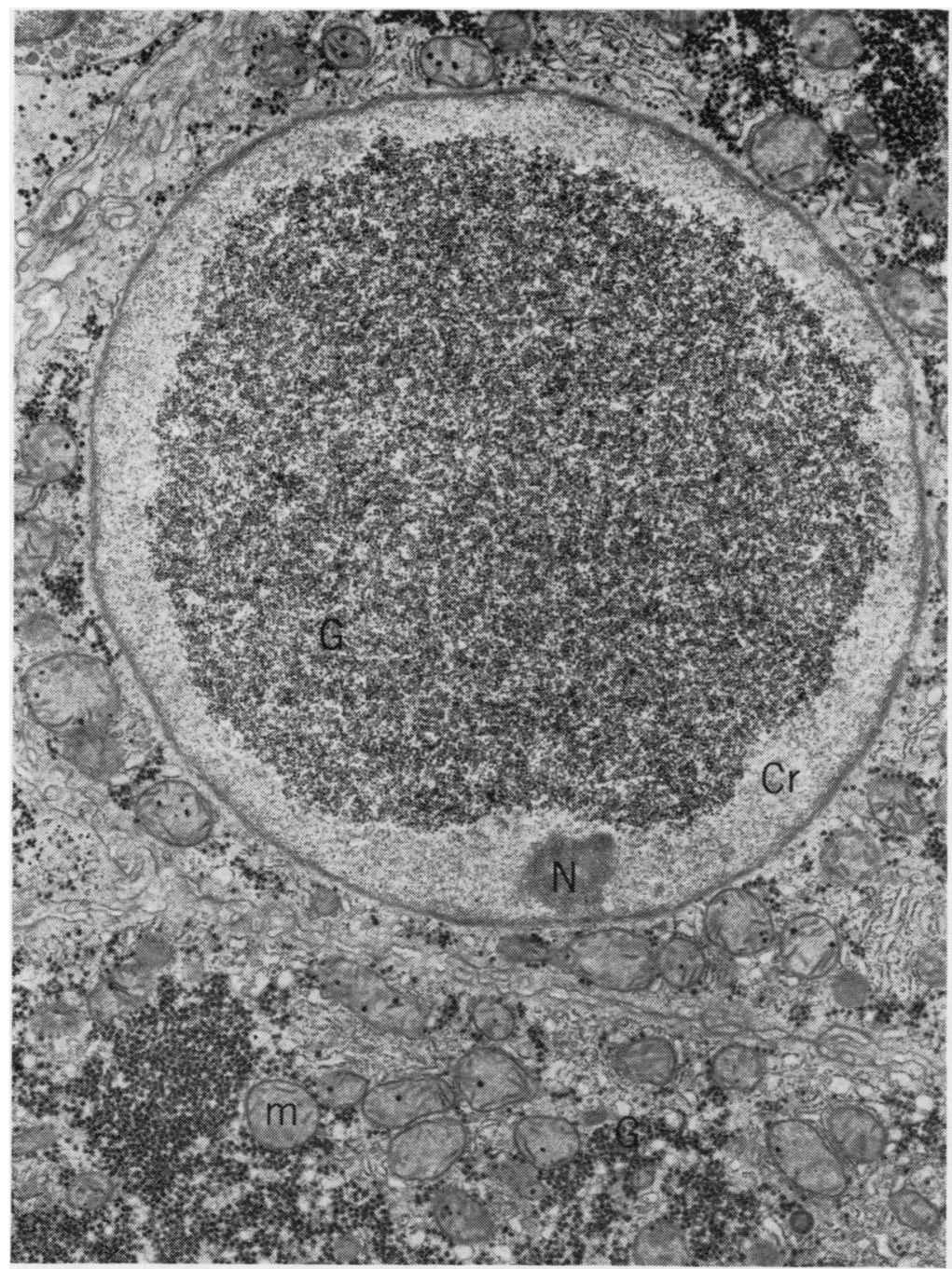

FIG. 3. This electron micrograph shows a nucleus with a large central inclusion of glycogen. The chromatin, which is displaced to the periphery, entirely surrounds the glycogen mass in such a way that glycogen does not contact the double limiting membrane of the nucleus. No changes are observed in the cytoplasm where mitochondria and other organelles appear normal. Note the different structure of nuclear glycogen and cytoplasmic glycogen rosettes. Mitochondria $(M)$, glycogen $(G)$, Nucleolus $(N)$, Chromatin $(C r)$. $\times 7,300$.

nuclear membrane. Nuclear glycogen in our micrographs appeared in a monoparticulate form (250 $\AA$ ), occasionally disposed in a file of several particles but even in large deposits never in the form of a rosette. In early stages nuclear glycogen particles tended to assume a rosette-like form but were larger than cytoplasmic rosettes. In our material we were unable to confirm the observations of Biava (1963) and Sparrow and Ashworth (1965) that the nuclear glycogen particle (about $250 \AA$ in diameter) is composed of subunits.

In many hepatocyte nuclei, in addition to the large glycogen inclusion, there was an intimately associated dense body. The available evidence suggests that these round bodies also contain glycogen, i.e., because lead staining increased the density of the central part and because they were P.A.S.-positive. Assuming that the association of these bodies with the nuclear glycogen is not incidental, our findings seem compatible with the hypothesis that such round bodies precede the deposition of glycogen. It is rare to find early forms of glycogenosis without this round body, whereas it is frequently possible to find the isolated round body without glycogen. In addition early glycogen deposits are localized in the immediate vicinity of the halo which surrounds the dense body, but only on one side. This may account for the fact that when the nucleus is filled with glycogen such dense bodies are located, as is the nucleolus, at the periphery of the nucleus, rather than being embedded within the glycogen mass. We do 


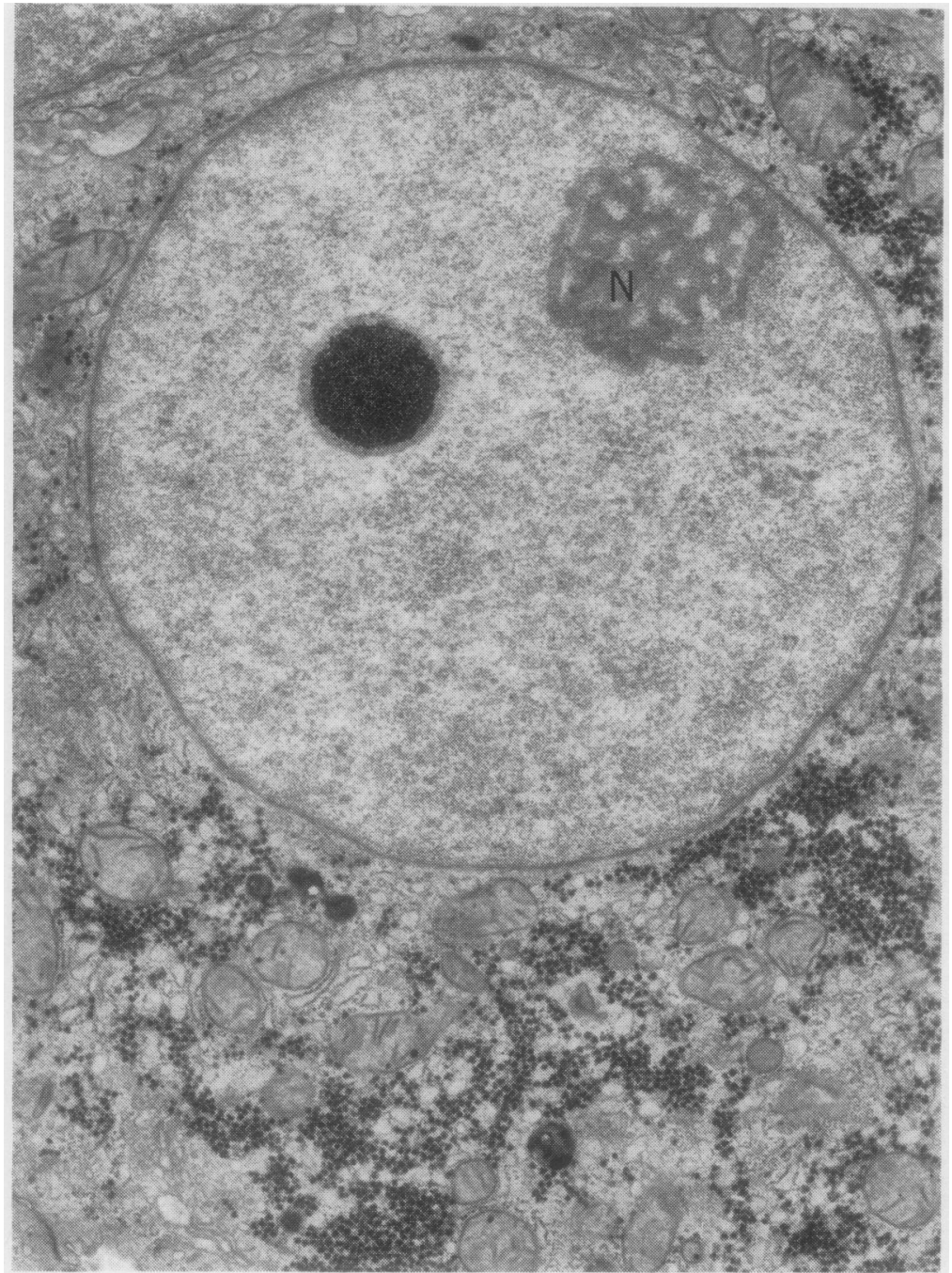

FIG. 4. A round dense body is present in the nucleus. It consists of a central mass of particulate material surrounded by a relatively pale halo $(\mathrm{Nu}) . \times 8,000$.

not have any explanation for these two different forms in which nuclear glycogen appears. One possibility is that the dense body represents an attempt by the nucleus to encapsulate early stages of glycogen formation. On the other hand the fact that we found these bodies in a high percentage of nuclei containing glycogen and only one per nucleus suggests that the dense body represents an indispensable step in the formation of nuclear glycogen.

Regarding the pathogenesis of such lesions, although some cases have been observed after intense treatment with corticosteroids (Sparrow and Ashworth, 1965), the majority of cases have not been associated with these drugs. We do not believe that the presence of nuclear glycogenosis in patients with protracted disease is necessarily due to stress with ? subsequent release of endogenous corticosteroids $N$ and increased gluconeogenesis. This could be true in ${ }_{\mathrm{C}}$ cases with increased cytoplasmic as well as nuclear 0 glycogen, as in the case of Sparrow. The cytoplasmic glycogen in the three cases we have observed did not $\frac{\vec{\Phi}}{\Phi}$ appear to be increased; some cells with nuclear $\stackrel{9}{+}$ glycogenosis had relatively scarce cytoplasmic 0 glycogen. We have observed the dense bodies in every case of nuclear glycogenosis so far examined, in the $\mathbb{\perp}$ three cases reported here, and in a fourth non-diabetic $\overrightarrow{\mathbb{D}}$ patient to be the subject of a separate report. We have no explanation for the failure of numerous 
